\title{
Some Properties of Complex Fuzzy Integral
}

\author{
Cuilian You and Weiqing Wang \\ College of Mathematics and Information Science, Hebei University, Baoding 071002, China \\ Correspondence should be addressed to Cuilian You; yycclian@163.com
}

Received 28 December 2014; Revised 16 April 2015; Accepted 17 April 2015

Academic Editor: Mohamed Abd El Aziz

Copyright (C) 2015 C. You and W. Wang. This is an open access article distributed under the Creative Commons Attribution License, which permits unrestricted use, distribution, and reproduction in any medium, provided the original work is properly cited.

In order to simplify the calculations of complex fuzzy integral (complex Liu integral), this paper aims to discuss the properties of this kind of complex fuzzy integral. Firstly, some properties of one-dimensional complex fuzzy integral are given. Then the properties above are extended to the case of multidimensional complex fuzzy integral.

\section{Introduction}

Many problems in the world are hard to be described statically. To deal with dynamic fuzzy phenomena, Liu [1] proposed the concept of fuzzy process with credibility measure. A detailed survey on credibility theory may be found in Liu [2]. As for more concepts and results, interested reader may consult Liu [3]. Subsequently, Liu process, Liu integral, and Liu formula were presented by Liu [1], which are the bases of fuzzy calculus. Later some researches surrounding Liu process have been done. You et al. [4] studied multidimensional Liu process, Liu formula, and Liu integral. Some properties of Liu integral were discussed by You and Wang [5], then these properties were extended to multidimensional Liu integral by You and Huo [6]. Lipschitz continuity of Liu process was proved by Dai [7]. Besides, You et al. [8] derived existence and uniqueness theorems for two special fuzzy differential equations. Chen and Qin [9] extended the existence and uniqueness theorem to the general fuzzy differential equations.

As for applications in finance, to describe the variance of the price of a stock in fuzzy market, fuzzy differential equation is an important tool. Based on Liu process, Liu [1] initiated a stock model named Liu's stock model. Considering that the price of a stock is a solution of a fuzzy differential equation, Qin and Li [10] deduced option pricing formula for European option. Other kinds of stock model were presented by Gao [11] and Peng [12]. Applying the knowledge of fuzzy differential equations, Qin and $\mathrm{Li}$ [13] studied the problems of trading strategies. While in the solving process of fuzzy differential equation, fuzzy integral (Liu integral) is needed. As for applications in control, it is known that classical control system is described by a differential equation, if there exist some fuzzy factors, fuzzy differential equations must be taken into consideration. Based on Liu process, Zhu [14] gave the optimal equation in the field of fuzzy control. Qin et al. [15] applied the fuzzy control to production planning problem.

As we know, there exist complex stochastic processes in many fields, such as signal analysis, thermodynamics, and fluid dynamics. Complex stochastic process is different from the two-dimensional stochastic process since it can reveal the relation of its real and imaginary parts. However, if the uncertainty factor is dominated by fuzzy factors in the above systems, how can we deal with them? In order to answer this question, Yang [16] defined a complex fuzzy variable whose real and imaginary parts are both fuzzy variables. Similarly the complex fuzzy process is introduced. In particular, the properties of complex fuzzy process were given by Qin and Wen [17].

Despite the importance of complex fuzzy process, there are so few literatures on this topic, considering these reasons, in this paper, based on credibility theory, the properties of complex fuzzy integral will be presented. For this purpose, the paper is organized as follows: Some basic definitions and properties of Liu integral and the definitions of complex fuzzy process are recalled in Section 2. Section 3 demonstrates the properties of one-dimensional complex fuzzy integral. The definition of $m$-dimensional complex fuzzy integral is given in Section 4, then some properties are obtained. Finally a brief conclusion is given in Section 5 . 


\section{Preliminaries}

In this section, some basic definitions and properties of credibility theory are recalled.

Let $\Theta$ be a nonempty set and $\mathscr{P}$ the power set of $\Theta$. For any $A \in \mathscr{P}$, a credibility measure $\operatorname{Cr}\{A\}$ describes the chance that fuzzy event occurs. In 2006, Liu [3] proved that a set function $\mathrm{Cr}$ is a credibility measure if and only if

(1) $\operatorname{Cr}\{\Theta\}=1$;

(2) $\operatorname{Cr}\{A\} \leq \operatorname{Cr}\{B\}$, whenever $A \subset B$;

(3) $\operatorname{Cr}\{A\}+\operatorname{Cr}\left\{A^{c}\right\}=1$, for any $A \in \mathscr{P}$;

(4) $\operatorname{Cr}\left\{\cup_{i} A_{i}\right\}=\sup _{i} \operatorname{Cr}\left\{A_{i}\right\}$ for any collection $A_{i}$ in $\mathscr{P}$ with $\sup _{i} \operatorname{Cr}\left\{A_{i}\right\}<0.5$. [3].

The triplet $(\Theta, \mathscr{P}, \mathrm{Cr})$ is called a credibility space in Liu

Definition 1 (Liu [1]). A fuzzy process $C_{t}$ is said to be a Liu process if

(1) $C_{0}=0$,

(2) $C_{t}$ has stationary and independent increments,

(3) every increment $C_{s+t}-C_{s}$ is a normally distributed fuzzy variable with expected value et and variance $\sigma^{2} t^{2}$, whose membership function is

$$
\mu(x)=2\left(1+\exp \left(\frac{\pi|x-e t|}{\sqrt{6} t}\right)\right)^{-1}, \quad-\infty<x<\infty .
$$

The parameters $e$ and $\sigma$ are called drift and diffusion coefficients, respectively. Liu process is said to be standard if $e=0$ and $\sigma=1$.

Definition 2 (Liu [1]). Let $X_{t}$ be a fuzzy process and let $C_{t}$ be a standard Liu process. For any partition of closed interval $[a, b]$ with $a=t_{1}<t_{2} \cdots<t_{k+1}=b$, the mesh is written as $\Delta=\max _{1 \leq i \leq k}\left|t_{p+1}-t_{p}\right|$. Then the Liu integral of $X_{t}$ with respect to $C_{t}$ is

$$
\int_{a}^{b} X_{t} \mathrm{~d} C_{t}=\lim _{\Delta \rightarrow 0} \sum_{p=1}^{k} X_{t p}\left(C_{t_{p+1}}-C_{t_{p}}\right)
$$

provided that the limit exists almost surely and is a fuzzy variable.

Theorem 3 (You and Wang [5]). Let fuzzy process $X_{t}$ be monotonous and bounded with respect to $t$. Then Liu integral $\int_{a}^{b} X_{t} \mathrm{~d} C_{t}$ exists.

Theorem 4 (You and Wang [5]). Let $X_{t}$ be a continuous fuzzy process. Then integral $\int_{a}^{b} X_{t} \mathrm{~d} C_{t}$ exists.

Theorem 5 (You and Wang [5]). Let $a<k<b$. Iffuzzy process $X_{t}$ is Liu integrable on any closed interval $[a, k]$ and $[k, b]$, then $X_{t}$ is integrable on closed interval $[a, b]$, and

$$
\int_{a}^{b} X_{t} \mathrm{~d} C_{t}=\int_{a}^{k} X_{t} \mathrm{~d} C_{t}+\int_{k}^{b} X_{t} \mathrm{~d} C_{t}
$$

Theorem 6 (You and Huo [6]). If $X_{t}, Y_{t}$ are monotonous and bounded fuzzy processes, then $\int_{a}^{b} X_{t} Y_{t} \mathrm{~d} C_{t}$ exists.

Definition 7 (Qin and Wen [17]). Let $T$ be an index set and let $(\Theta, \mathscr{P}, \mathrm{Cr})$ be a credibility space. A complex fuzzy process is a function from $T \times(\Theta, \mathscr{P}, \mathrm{Cr})$ to the set of complex numbers.

Definition 8 (Qin and Wen [17]). If $C_{1 t}$ and $C_{2 t}$ are independent Liu processes, then $C_{t}=C_{1 t}+i C_{2 t}$ is called a complex Liu process. Here $i$ is an imaginary number.

Theorem 9 (Qin and Wen [17]). A process $X_{t}$ is a complex fuzzy process if and only if there exist two fuzzy processes $X_{1 t}$ and $X_{2 t}$ such that $X_{t}=X_{1 t}+i X_{2 t}$.

Definition 10 (Qin and Wen [17]). Let $X_{t}=X_{1 t}+i X_{2 t}$ be a complex fuzzy process and let $C_{t}=C_{1 t}+i C_{2 t}$ be a standard complex Liu process. Then the complex fuzzy integral of $X_{t}$ with respect to $C_{t}$ is defined by

$$
\begin{aligned}
\int_{a}^{b} X_{t} \mathrm{~d} C_{t}= & \int_{a}^{b}\left(X_{1 t} \mathrm{~d} C_{1 t}-X_{2 t} \mathrm{~d} C_{2 t}\right) \\
& +i \int_{a}^{b}\left(X_{1 t} \mathrm{~d} C_{2 t}+X_{2 t} \mathrm{~d} C_{1 t}\right) .
\end{aligned}
$$

We will discuss some properties of complex fuzzy integral in next two sections, which will be useful for the calculations of fuzzy integral.

\section{One-Dimensional Complex Fuzzy Integral}

Theorem 11. If $X_{t}=X_{1 t}+i X_{2 t}$ is a complex fuzzy process, $X_{1 t}$ and $X_{2 t}$ are two continuous fuzzy processes, and $C_{t}=C_{1 t}+i C_{2 t}$ is a complex Liu process, then Liu integral $\int_{a}^{b} X_{t} \mathrm{~d} C_{t}$ exists.

Proof. It follows from the definition of complex Liu integral that

$$
\begin{aligned}
\int_{a}^{b} X_{t} \mathrm{~d} C_{t}= & \int_{a}^{b}\left(X_{1 t}+i X_{2 t}\right) \mathrm{d}\left(C_{1 t}+i C_{2 t}\right) \\
= & \int_{a}^{b} X_{1 t}\left(\mathrm{~d} C_{1 t}+i \mathrm{~d}_{2 t}\right) \\
& +\int_{a}^{b} i X_{2 t}\left(\mathrm{~d} C_{1 t}+i \mathrm{~d} C_{2 t}\right) \\
= & \int_{a}^{b} X_{1 t} \mathrm{~d} C_{1 t}+i \int_{a}^{b} X_{1 t} \mathrm{~d} C_{2 t}+i \int_{a}^{b} X_{2 t} \mathrm{~d} C_{1 t} \\
& -\int_{a}^{b} X_{2 t} \mathrm{~d} C_{2 t} .
\end{aligned}
$$

According to Theorem $4, \int_{a}^{b} X_{1 t} \mathrm{~d} C_{1 t}, \int_{a}^{b} X_{1 t} \mathrm{~d} C_{2 t}, \int_{a}^{b} i X_{2 t} \mathrm{~d} C_{1 t}$, and $\int_{a}^{b} X_{2 t} \mathrm{~d} C_{2 t}$ exist and are finite, then Liu integral $\int_{a}^{b} X_{t} \mathrm{~d} C_{t}$ exists.

Theorem 12. Let $X_{t}$ be a complex fuzzy process and $X_{t}=X_{1 t}+$ $i X_{2 t}$, where $X_{1 t}$ and $X_{2 t}$ are monotonous and bounded with 
respect to $t$. Assume that $C_{t}=C_{1 t}+i C_{2 t}$ and $C_{1 t}, C_{2 t}$ are both standard Liu processes. Then integral $\int_{a}^{b} X_{t} \mathrm{~d} C_{t}$ exists.

Proof. It follows from the definition of complex Liu integral that

$$
\begin{aligned}
\int_{a}^{b} X_{t} \mathrm{~d} C_{t}= & \int_{a}^{b} X_{1 t} \mathrm{~d} C_{1 t}-\int_{a}^{b} X_{2 t} \mathrm{~d} C_{2 t}+i \int_{a}^{b} X_{2 t} \mathrm{~d} C_{1 t} \\
& +i \int_{a}^{b} X_{1 t} \mathrm{~d} C_{2 t},
\end{aligned}
$$

and by using Theorem 3, we know $\int_{a}^{b} X_{1 t} \mathrm{~d} C_{1 t}, \int_{a}^{b} X_{1 t} \mathrm{~d} C_{2 t}$, $\int_{a}^{b} i X_{2 t} \mathrm{~d} C_{1 t}$, and $\int_{a}^{b} X_{2 t} \mathrm{~d} C_{2 t}$ exist and are finite. Thus Liu integral $\int_{a}^{b} X_{t} \mathrm{~d} C_{t}$ exists.

Theorem 13. Suppose that $X_{t}=X_{1 t}+i X_{2 t}$ is Liu integrable complex fuzzy process and $C_{t}=C_{1 t}+i C_{2 t}$ is a complex Liu process. Then

$$
\int_{a}^{b} k X_{t} \mathrm{~d} C_{t}=k \int_{a}^{b} X_{t} \mathrm{~d} C_{t}
$$

for any complex number $k$.

Proof. It follows from Definition 8 that

$$
\begin{aligned}
& \int_{a}^{b} k X_{t} \mathrm{~d} C_{t}=\int_{a}^{b}\left(k X_{1 t}+i k X_{2 t}\right) \mathrm{d}\left(C_{1 t}+i C_{2 t}\right) \\
& =\int_{a}^{b} k X_{1 t} \mathrm{~d} C_{1 t}+\int_{a}^{b} i k X_{2 t} \mathrm{~d} C_{1 t}+\int_{a}^{b} i k X_{1 t} \mathrm{~d} C_{2 t} \\
& +\int_{a}^{b} i k X_{2 t} \mathrm{~d} i C_{2 t}=k \int_{a}^{b} X_{1 t} \mathrm{~d} C_{1 t}+i k \int_{a}^{b} X_{2 t} \mathrm{~d} C_{1 t} \\
& +i k \int_{a}^{b} X_{1 t} \mathrm{~d} C_{2 t}-k \int_{a}^{b} X_{2 t} \mathrm{~d} C_{2 t}=k\left(\int_{a}^{b} X_{1 t} \mathrm{~d} C_{1 t}\right. \\
& \left.-\int_{a}^{b} X_{2 t} \mathrm{~d} C_{2 t}+i \int_{a}^{b} i X_{2 t} \mathrm{~d} C_{1 t}+i \int_{a}^{b} X_{1 t} \mathrm{~d} C_{2 t}\right) \\
& =k \int_{a}^{b} X_{t} \mathrm{~d} C_{t} .
\end{aligned}
$$

The theorem is proved.

Theorem 14. Suppose that $X_{t}=X_{1 t}+i X_{2 t}, Y_{t}=Y_{1 t}+i Y_{2 t}$ are Liu integrable complex fuzzy processes and $C_{t}=C_{1 t}+i C_{2 t}$ is a complex Liu process. Then

$$
\int_{a}^{b}\left(X_{t}+Y_{t}\right) \mathrm{d} C_{t}=\int_{a}^{b} X_{t} \mathrm{~d} C_{t}+\int_{a}^{b} Y_{t} \mathrm{~d} C_{t} .
$$

Proof. It follows from the definition of complex Liu integral that

$$
\begin{aligned}
& \int_{a}^{b}\left(X_{t}+Y_{t}\right) \mathrm{d} C_{t} \\
& \quad=\int_{a}^{b}\left(\left(X_{1 t}+Y_{1 t}\right)+i\left(X_{2 t}+Y_{2 t}\right)\right) \mathrm{d} C_{t}
\end{aligned}
$$

$$
\begin{aligned}
= & \int_{a}^{b}\left(X_{1 t}+Y_{1 t}\right) \mathrm{d} C_{t}+i \int_{a}^{b}\left(X_{2 t}+Y_{2 t}\right) \mathrm{d} C_{t} \\
= & \int_{a}^{b} X_{1 t} \mathrm{~d} C_{t}+\int_{a}^{b} Y_{1 t} \mathrm{~d} C_{t}+i \int_{a}^{b} X_{2 t} \mathrm{~d} C_{t} \\
& +i \int_{a}^{b} Y_{2 t} \mathrm{~d} C_{t} \\
= & \int_{a}^{b} X_{1 t} \mathrm{~d} C_{t}+i \int_{a}^{b} X_{2 t} \mathrm{~d} C_{t}+\int_{a}^{b} Y_{1 t} \mathrm{~d} C_{t} \\
& +i \int_{a}^{b} Y_{2 t} \mathrm{~d} C_{t}=\int_{a}^{b} X_{t} \mathrm{~d} C_{t}+\int_{a}^{b} Y_{t} \mathrm{~d} C_{t} .
\end{aligned}
$$

The proof is completed.

Remark 15. It follows from Theorems 13 and 14 that

$$
\int_{a}^{b}\left(k_{1} X_{t}+k_{2} Y_{t}\right) \mathrm{d} C_{t}=k_{1} \int_{a}^{b} X_{t} \mathrm{~d} C_{t}+k_{2} \int_{a}^{b} Y_{t} \mathrm{~d} C_{t},
$$

for any complex numbers $k_{1}$ and $k_{2}$.

Theorem 16. If $X_{t}=X_{1 t}+i X_{2 t}, Y_{t}=Y_{1 t}+i Y_{2 t}$, where $X_{1 t}$, $X_{2 t}, Y_{1 t}$, and $Y_{2 t}$ are monotonous and bounded fuzzy processes, and $C_{t}=C_{1 t}+i C_{2 t}$ is a complex Liu process, then $\int_{a}^{b} X_{t} Y_{t} \mathrm{~d} C_{t}$ exists.

Proof. It follows from the definition of complex Liu integral that

$$
\begin{aligned}
\int_{a}^{b} & \left(X_{t} Y_{t}\right) \mathrm{d} C_{t} \\
= & \int_{a}^{b}\left(\left(X_{1 t} Y_{1 t}-X_{2 t} Y_{2 t}\right)+i\left(X_{1 t} Y_{2 t}+X_{2 t} Y_{1 t}\right)\right) \mathrm{d} C_{t} \\
= & \int_{a}^{b}\left(X_{1 t} Y_{1 t}-X_{2 t} Y_{2 t}\right) \mathrm{d} C_{1 t} \\
& -\int_{a}^{b}\left(X_{1 t} Y_{2 t}+X_{2 t} Y_{1 t}\right) \mathrm{d} C_{2 t} \\
& +i \int_{a}^{b}\left(X_{1 t} Y_{1 t}-X_{2 t} Y_{2 t}\right) \mathrm{d} C_{2 t} \\
& +i \int_{a}^{b}\left(X_{1 t} Y_{2 t}+X_{2 t} Y_{1 t}\right) \mathrm{d} C_{1 t} \\
= & \int_{a}^{b} X_{1 t} Y_{1 t} \mathrm{~d} C_{1 t}-\int_{a}^{b} X_{2 t} Y_{2 t} \mathrm{~d} C_{1 t}-\int_{a}^{b} X_{1 t} Y_{2 t} \mathrm{~d} C_{2 t} \\
& -\int_{a}^{b} X_{2 t} Y_{1 t} \mathrm{~d} C_{2 t}+i \int_{a}^{b} X_{1 t} Y_{1 t} \mathrm{~d} C_{2 t} \\
& +i \int_{a}^{b} \int_{a}^{b} X_{2 t} Y_{2 t} \mathrm{~d} C_{2 t}+i \int_{a}^{b} X_{1 t} Y_{2 t} \mathrm{~d} C_{1 t},
\end{aligned}
$$


and by using Theorem 6, we have $\int_{a}^{b} X_{1 t} Y_{1 t} \mathrm{~d} C_{1 t}$, $\int_{a}^{b} X_{2 t} Y_{2 t} \mathrm{~d} C_{1 t}, \int_{a}^{b} X_{1 t} Y_{2 t} \mathrm{~d} C_{2 t}, \int_{a}^{b} X_{2 t} Y_{1 t} \mathrm{~d} C_{2 t}, \int_{a}^{b} X_{1 t} Y_{1 t} \mathrm{~d} C_{2 t}$, $\int_{a}^{b} X_{2 t} Y_{2 t} \mathrm{~d} C_{2 t}, \int_{a}^{b} X_{1 t} Y_{2 t} \mathrm{~d} C_{1 t}$, and $\int_{a}^{b} X_{2 t} Y_{1 t} \mathrm{~d} C_{1 t}$ exist. Hence $\int_{a}^{b} X_{t} Y_{t} \mathrm{~d} C_{t}$ exists.

Theorem 17. Suppose that $a<k<b$. Let $X_{t}=X_{1 t}+i X_{2 t}$ be a Liu integrable complex fuzzy process and $C_{t}=C_{1 t}+i C_{2 t} a$ complex Liu process. Then

$$
\int_{a}^{b} X_{t} \mathrm{~d} C_{t}=\int_{a}^{k} X_{t} \mathrm{~d} C_{t}+\int_{k}^{b} X_{t} \mathrm{~d} C_{t}
$$

Proof. It follows from the definition of complex Liu integral and Theorem 5 that

$$
\begin{aligned}
\int_{a}^{b} X_{t} \mathrm{~d} C_{t}= & \int_{a}^{b} X_{1 t} \mathrm{~d} C_{1 t}-\int_{a}^{b} X_{2 t} \mathrm{~d} C_{2 t}+i \int_{a}^{b} X_{2 t} \mathrm{~d} C_{1 t} \\
& +i \int_{a}^{b} X_{1 t} \mathrm{~d} C_{2 t} \\
= & \int_{a}^{k} X_{1 t} \mathrm{~d} C_{1 t}+\int_{k}^{b} X_{1 t} \mathrm{~d} C_{1 t} \\
& -\left(\int_{a}^{k} X_{2 t} \mathrm{~d} C_{2 t}+\int_{k}^{b} X_{2 t} \mathrm{~d} C_{2 t}\right) \\
& +i\left(\int_{a}^{k} X_{2 t} \mathrm{~d} C_{1 t}+\int_{k}^{b} X_{2 t} \mathrm{~d} C_{1 t}\right) \\
& +i\left(\int_{a}^{k} X_{1 t} \mathrm{~d} C_{2 t}+\int_{k}^{b} X_{1 t} \mathrm{~d} C_{2 t}\right) \\
= & \int_{a}^{k} X_{t} \mathrm{~d} C_{t}+\int_{b}^{k} X_{t} \mathrm{~d} C_{t} .
\end{aligned}
$$

The theorem holds.

\section{Multidimensional Complex Fuzzy Integral}

Definition 18. If $C_{p t}, p=1,2, \ldots, m$, are complex Liu processes, then $\mathbf{C}_{t}=\left(C_{1 t}, C_{2 t}, \ldots, C_{m t}\right)^{T}$ is called an $m$-dimensional complex Liu process.

In particular, if $C_{p t}, p=1,2, \ldots, m$, are standard complex Liu processes, $\mathbf{C}_{t}=\left(C_{1 t}, C_{2 t}, \ldots, C_{m t}\right)^{T}$ is called an $m$ dimensional standard complex Liu process.

Definition 19. Let $\mathbf{C}_{t}=\left(C_{1 t}, C_{2 t}, \ldots, C_{m t}\right)^{T}$ be an $m$ dimensional standard complex Liu process. Denote by $\mathscr{V}^{n \times m}$, the set of $n \times m$ matrices $v_{t}=\left[v_{p q t}\right]$, where each entry $v_{p q t}$ is a Liu integrable complex fuzzy process, $v_{p q t}=v_{p q 1 t}+i v_{p q 2 t}$, $C_{p t}=C_{p 1 t}+i C_{p 2 t}$. Suppose $a<b$. If $v_{t} \in \mathscr{V}^{m \times n}$, then the $m$-dimensional complex Liu integral is defined, using matrix notation

$$
\int_{a}^{b} v_{t} \mathrm{dC}_{t}=\int_{a}^{b}\left(\begin{array}{cccc}
v_{11 t} & v_{12 t} & \cdots & v_{1 m t} \\
v_{21 t} & v_{22 t} & \cdots & v_{2 m t} \\
\vdots & \vdots & & \vdots \\
v_{n 1 t} & v_{n 2 t} & \cdots & v_{n m t}
\end{array}\right)\left(\begin{array}{c}
\mathrm{d}_{1 t} \\
\mathrm{~d}_{2 t} \\
\vdots \\
\mathrm{d} C_{m t}
\end{array}\right)
$$

as the $n \times 1$ matrix whose $p$ th component is the following sum of complex Liu integral

$$
\begin{aligned}
& \sum_{q=1}^{m} \int_{a}^{b} v_{p q t} \mathrm{~d} C_{q t}=\sum_{q=1}^{m}\left(\int_{a}^{b} v_{p q 1 t} \mathrm{~d} C_{q 1 t}+i \int_{a}^{b} v_{p q 1 t} \mathrm{~d} C_{q 2 t}\right. \\
& \left.+i \int_{a}^{b} v_{p q 2 t} \mathrm{~d} C_{q 1 t}-\int_{a}^{b} v_{p q 2 t} \mathrm{~d} C_{q 2 t}\right) .
\end{aligned}
$$

In this case, $v_{t}$ is called a complex Liu integrable matrix.

Example 20. Let $C_{11 t}, C_{12 t}, C_{21 t}, C_{22 t}, C_{23 t}$ be Liu processes and $\mathbf{C}_{t}=\left(C_{1 t}, C_{2 t}\right)$, where $C_{i t}=C_{i 1 t}+i C_{i 2 t}, i=1,2$. Then

$$
v_{t}=\left(\begin{array}{cc}
t+i C_{11 t} & C_{12 t} \\
C_{21 t}+i t & C_{22 t}+i C_{23 t}
\end{array}\right)
$$

is a complex Liu integrable matrix, and

$$
\int_{a}^{b} v_{t} \mathrm{~d} \mathbf{C}_{t}=\left(\begin{array}{c}
m \\
n
\end{array}\right),
$$

where

$$
\begin{aligned}
m & =\int_{a}^{b} t \mathrm{~d} C_{11 t}-\int_{a}^{b} C_{11 t} \mathrm{~d} C_{12 t}+\int_{a}^{b} C_{12 t} \mathrm{~d} C_{11 t} \\
& +i\left(\int_{a}^{b} t \mathrm{~d} C_{12 t}+\int_{a}^{b} C_{11 t} \mathrm{~d} C_{11 t}+\int_{a}^{b} C_{12 t} \mathrm{~d} C_{12 t}\right), \\
n & =\int_{a}^{b} C_{21 t} \mathrm{~d} C_{11 t}-\int_{a}^{b} t \mathrm{~d} C_{12 t}+\int_{a}^{b} C_{22 t} \mathrm{~d} C_{21 t} \\
& -\int_{a}^{b} C_{23 t} \mathrm{~d} C_{22 t}+i\left(\int_{a}^{b} C_{21 t} \mathrm{~d} C_{12 t}+\int_{a}^{b} t \mathrm{~d} C_{11 t}\right. \\
& \left.+\int_{a}^{b} C_{22 t} \mathrm{~d} C_{22 t}+\int_{a}^{b} C_{23 t} \mathrm{~d} C_{21 t}\right) .
\end{aligned}
$$

Theorem 21. Let $v_{t}=\left[v_{p q t}\right]$ be $n \times m$ complex Liu integrable matrix. Then $l v_{t}$ is complex integrable and

$$
\int_{a}^{b} l v_{t} \mathrm{~d} \mathbf{C}_{t}=l \int_{a}^{b} v_{t} \mathrm{~d} \mathbf{C}_{t}
$$

for any complex number $l$.

Proof. Since $v_{p q t}$ are complex Liu integrable fuzzy processes, for $p=1,2,3, \ldots, q=1,2,3, \ldots$, it follows from Theorem 13 that

$$
\int_{a}^{b} l v_{p q t} \mathrm{~d} C_{q t}=l \int_{a}^{b} v_{p q t} \mathrm{~d} C_{q t} .
$$


Mathematical Problems in Engineering

5

Hence $l v_{t}$ is complex Lu integrable matrix, and

$$
\begin{aligned}
& \int_{a}^{b} l v_{t} \mathrm{~d} \mathbf{C}_{t} \\
& =\int_{a}^{b}\left(\begin{array}{cccc}
l v_{11 t} & l v_{12 t} & \cdots & l v_{1 m t} \\
l v_{21 t} & l v_{22 t} & \cdots & l v_{2 m t} \\
\vdots & \vdots & & \vdots \\
l v_{n 1 t} & l v_{n 2 t} & \cdots & l v_{n m t}
\end{array}\right)\left(\begin{array}{c}
\mathrm{d}_{1 t} \\
\mathrm{~d}_{2 t} \\
\vdots \\
\mathrm{d} C_{m t}
\end{array}\right) \\
& =\left(\begin{array}{c}
\int_{a}^{b} l v_{11 t} \mathrm{~d} C_{1 t}+\int_{a}^{b} l v_{12 t} \mathrm{~d} C_{2 t}+\cdots+\int_{a}^{b} l v_{1 m t} \mathrm{~d} C_{m t} \\
\int_{a}^{b} l v_{21 t} \mathrm{~d} C_{1 t}+\int_{a}^{b} l v_{22 t} \mathrm{~d} C_{2 t}+\cdots+\int_{a}^{b} l v_{2 m t} \mathrm{~d} C_{m t} \\
\vdots \\
\int_{a}^{b} l v_{n 1 t} \mathrm{~d} C_{1 t}+\int_{a}^{b} l v_{n 2 t} \mathrm{~d} C_{2 t}+\cdots+\int_{a}^{b} l v_{n m t} \mathrm{~d} C_{m t}
\end{array}\right)
\end{aligned}
$$

$$
\begin{aligned}
& =\left(\begin{array}{c}
l\left(\int_{a}^{b} v_{11 t} \mathrm{~d} C_{1 t}+\int_{a}^{b} v_{12 t} \mathrm{~d} C_{2 t}+\cdots+\int_{a}^{b} v_{1 m t} \mathrm{~d} C_{m t}\right) \\
l\left(\int_{a}^{b} v_{21 t} \mathrm{~d} C_{1 t}+\int_{a}^{b} v_{22 t} \mathrm{~d} C_{2 t}+\cdots+\int_{a}^{b} v_{2 m t} \mathrm{~d} C_{m t}\right) \\
\vdots \\
l\left(\int_{a}^{b} v_{n 1 t} \mathrm{~d} C_{1 t}+\int_{a}^{b} v_{n 2 t} \mathrm{~d} C_{2 t}+\cdots+\int_{a}^{b} v_{n m t} \mathrm{~d} C_{m t}\right)
\end{array}\right) \\
& =l \int_{a}^{b} v_{t} \mathrm{dC}_{t} .
\end{aligned}
$$

The theorem holds.

Theorem 22. Let $u_{t}, v_{t} \in \mathscr{V}^{n \times \mathrm{m}}$ be complex Lin integrable matrices. Then $u_{t}+v_{t}$ is complex Lin integrable matrix, and

$$
\int_{a}^{b}\left(u_{t}+v_{t}\right) \mathrm{d} C_{t}=\int_{a}^{b} v_{t} \mathrm{~d} C_{t}+\int_{a}^{b} u_{t} \mathrm{~d} C_{t} .
$$

Proof. It follows from the definition of multidimensional complex Lin integral that

$$
\begin{aligned}
& \int_{a}^{b}\left(v_{t}+u_{t}\right) \mathrm{d} \mathbf{C}_{t}=\int_{a}^{b}\left(\begin{array}{cccc}
v_{11 t}+u_{11 t} & v_{12 t}+u_{12 t} & \cdots & v_{1 m t}+u_{1 m t} \\
v_{21 t}+u_{21 t} & v_{22 t}+u_{22 t} & \cdots & v_{2 m t}+u_{2 m t} \\
\vdots & \vdots & & \vdots \\
v_{n 1 t}+u_{n 1 t} & v_{n 2 t}+u_{n 2 t} & \cdots & v_{n m t}+u_{n m t}
\end{array}\right)\left(\begin{array}{c}
\mathrm{d} C_{1 t} \\
\mathrm{~d} C_{2 t} \\
\vdots \\
\mathrm{d} C_{m t}
\end{array}\right) \\
& =\left(\begin{array}{c}
\int_{a}^{b}\left(v_{11 t}+u_{11 t}\right) \mathrm{d} C_{1 t}+\int_{a}^{b}\left(v_{12 t}+u_{12 t}\right) \mathrm{d} C_{2 t}+\cdots+\int_{a}^{b}\left(v_{1 m t}+u_{1 m t}\right) \mathrm{d} C_{m t} \\
\int_{a}^{b}\left(v_{21 t}+u_{21 t}\right) \mathrm{d} C_{1 t}+\int_{a}^{b}\left(v_{22 t}+u_{22 t}\right) \mathrm{d} C_{2 t}+\cdots+\int_{a}^{b}\left(v_{2 m t}+u_{2 m t}\right) \mathrm{d} C_{m t} \\
\vdots \\
\int_{a}^{b}\left(v_{n 1 t}+u_{n 1 t}\right) \mathrm{d} C_{1 t}+\int_{a}^{b}\left(v_{n 2 t}+u_{n 2 t}\right) \mathrm{d} C_{2 t}+\cdots+\int_{a}^{b}\left(v_{n m t}+u_{n m t}\right) \mathrm{d} C_{m t}
\end{array}\right) \\
& =\left(\begin{array}{c}
\left(\int_{a}^{b} v_{11 t} \mathrm{~d} C_{1 t}+\cdots+\int_{a}^{b} v_{1 m t} \mathrm{~d} C_{m t}\right)+\left(\int_{a}^{b} u_{11 t} \mathrm{~d} C_{1 t}+\cdots+\int_{a}^{b} u_{1 m t} \mathrm{~d} C_{m t}\right) \\
\left(\int_{a}^{b} v_{21 t} \mathrm{~d} C_{1 t}+\cdots+\int_{a}^{b} v_{2 m t} \mathrm{~d} C_{m t}\right)+\left(\int_{a}^{b} u_{21 t} \mathrm{~d} C_{1 t}+\cdots+\int_{a}^{b} u_{2 m t} \mathrm{~d} C_{m t}\right) \\
\vdots \\
\left(\int_{a}^{b} v_{n 1 t} \mathrm{~d} C_{1 t}+\cdots+\int_{a}^{b} v_{n m t} \mathrm{~d} C_{m t}\right)+\int_{a}^{b} u_{n 1 t} \mathrm{~d} C_{1 t}+\cdots+\int_{a}^{b} u_{n m t} \mathrm{~d} C_{m t}
\end{array}\right) \\
& =\left(\begin{array}{c}
\int_{a}^{b} v_{11 t} \mathrm{~d} C_{1 t}+\int_{a}^{b} v_{12 t} \mathrm{~d} C_{2 t}+\cdots+\int_{a}^{b} v_{1 m t} \mathrm{~d} C_{m t} \\
\int_{a}^{b} v_{21 t} \mathrm{~d} C_{1 t}+\int_{a}^{b} v_{22 t} \mathrm{~d} C_{2 t}+\cdots+\int_{a}^{b} v_{2 m t} \mathrm{~d} C_{m t} \\
\vdots \\
\int_{a}^{b} v_{n 1 t} \mathrm{~d} C_{1 t}+\int_{a}^{b} v_{n 2 t} \mathrm{~d} C_{2 t}+\cdots+\int_{a}^{b} v_{n m t} \mathrm{~d} C_{m t}
\end{array}\right)
\end{aligned}
$$




$$
+\left(\begin{array}{c}
\int_{a}^{b} u_{11 t} \mathrm{~d} C_{1 t}+\int_{a}^{b} u_{12 t} \mathrm{~d} C_{2 t}+\cdots+\int_{a}^{b} u_{1 m t} \mathrm{~d} C_{m t} \\
\int_{a}^{b} u_{21 t} \mathrm{~d} C_{1 t}+\int_{a}^{b} u_{22 t} \mathrm{~d} C_{2 t}+\cdots+\int_{a}^{b} u_{2 m t} \mathrm{~d} C_{m t} \\
\vdots \\
\int_{a}^{b} u_{n 1 t} \mathrm{~d} C_{1 t}+\int_{a}^{b} u_{n 2 t} \mathrm{~d} C_{2 t}+\cdots+\int_{a}^{b} u_{n m t} \mathrm{~d} C_{m t}
\end{array}\right)=\int_{a}^{b} v_{t} \mathrm{dC}_{t}+\int_{a}^{b} u_{t} \mathrm{dC}_{t}
$$

Remark 23. Let $u_{t}, v_{t} \in \mathscr{V}^{n \times m}$ be complex Liu integrable matrices. Then $u_{t}-v_{t}$ is complex Liu integrable matrix, and

$$
\int_{a}^{b}\left(v_{t}-u_{t}\right) \mathrm{d} \mathbf{C}_{t}=\int_{a}^{b} v_{t} \mathrm{~d} \mathbf{C}_{t}-\int_{a}^{b} u_{t} \mathrm{~d}_{t} .
$$

Remark 24. Let $u_{t}, v_{t} \in \mathscr{V}^{n \times m}$ be complex Liu integrable matrices. Then $k_{1} u_{t}+k_{2} v_{t}$ is complex Liu integrable matrix, and

$$
\int_{a}^{b}\left(k_{1} v_{t}+k_{2} u_{t}\right) \mathrm{d} \mathbf{C}_{t}=k_{1} \int_{a}^{b} v_{t} \mathrm{~d}_{t}+k_{2} \int_{a}^{b} u_{t} \mathrm{dC}_{t}
$$

for any complex numbers $k_{1}$ and $k_{2}$.
Theorem 25. Suppose that $a<k<b$. Let $v_{t} \in \mathscr{V}^{n \times m}$ denote the set of $n \times m$ complex matrices $v_{t}=\left[v_{p q t}\right]$, where each entry $v_{\text {pqt }}$ is Liu integrable complex fuzzy process. If $v_{t}$ is Liu integrable on $[a, k]$ and $[k, b]$, then $v_{t}$ is Liu integrable on $[a, b]$, and

$$
\int_{a}^{b} v_{t} \mathrm{~d}_{t}=\int_{a}^{k} v_{t} \mathrm{~d} \mathbf{C}_{t}+\int_{k}^{b} v_{t} \mathrm{dC}_{t}
$$

Proof. Since $v_{t}$ is Liu integrable on intervals $[a, k]$ and $[k, b]$, we have

$$
\begin{aligned}
\int_{a}^{k} v_{t} \mathrm{~d} \mathbf{C}_{t} & =\int_{a}^{k}\left(\begin{array}{cccc}
v_{11 t} & v_{12 t} & \cdots & v_{1 m t} \\
v_{21 t} & v_{22 t} & \cdots & v_{2 m t} \\
\vdots & \vdots & & \vdots \\
v_{n 1 t} & v_{n 2 t} & \cdots & v_{n m t}
\end{array}\right)\left(\begin{array}{c}
\mathrm{d} C_{1 t} \\
\mathrm{~d} C_{2 t} \\
\vdots \\
\mathrm{d} C_{m t}
\end{array}\right) \\
\int_{k}^{b} v_{t} \mathrm{~d} \mathbf{C}_{t}= & \int_{k}^{b}\left(\begin{array}{cccc}
v_{11 t} & v_{12 t} & \cdots & v_{1 m t} \\
v_{21 t} & v_{22 t} & \cdots & v_{2 m t} \\
\vdots & \vdots & & \vdots \\
\mathrm{d} C_{1 t} \\
\mathrm{~d} C_{2 t} \\
v_{n 1 t} & v_{n 2 t} & \cdots & v_{n m t}
\end{array}\right) \\
\int_{a}^{k} v_{t} \mathrm{~d} \mathbf{C}_{t}+\int_{k}^{b} v_{t} \mathrm{~d} \mathbf{C}_{t}= & \int_{a}^{k}\left(\begin{array}{c}
v_{11 t} \mathrm{~d} C_{1 t}+v_{12 t} \mathrm{~d} C_{2 t}+\cdots+v_{1 m t} \mathrm{~d} C_{m t} \\
v_{21 t} \mathrm{~d} C_{1 t}+v_{22 t} \mathrm{~d} C_{2 t}+\cdots+v_{2 m t} \mathrm{~d} C_{m t} \\
\vdots \\
v_{n 1 t} \mathrm{~d} C_{1 t}+v_{n 2 t} \mathrm{~d} C_{2 t}+\cdots+v_{n m t} \mathrm{~d} C_{m t}
\end{array}\right) \\
+ & \int_{k}^{b}\left(\begin{array}{c}
v_{11 t} \mathrm{~d} C_{1 t}+v_{12 t} \mathrm{~d} C_{2 t}+\cdots+v_{1 m t} \mathrm{~d} C_{m t} \\
v_{21 t} \mathrm{~d} C_{1 t}+v_{22 t} \mathrm{~d} C_{2 t}+\cdots+v_{2 m t} \mathrm{~d} C_{m t} \\
v_{n 1 t} \mathrm{~d} C_{1 t}+v_{n 2 t} \mathrm{~d} C_{2 t}+\cdots+v_{n m t} \mathrm{~d} C_{m t}
\end{array}\right)
\end{aligned}
$$




$$
=\left(\begin{array}{c}
\left(\int_{a}^{k} v_{11 t} \mathrm{~d} C_{1 t}+\int_{k}^{b} v_{11 t} \mathrm{~d} C_{1 t}\right)+\cdots+\left(\int_{a}^{k} v_{1 m t} \mathrm{~d} C_{m t}+\int_{k}^{b} v_{1 m t} \mathrm{~d} C_{m t}\right) \\
\left(\int_{a}^{k} v_{21 t} \mathrm{~d} C_{1 t}+\int_{k}^{b} v_{21 t} \mathrm{~d} C_{1 t}\right)+\cdots+\left(\int_{a}^{b} v_{2 m t} \mathrm{~d} C_{m t}+\int_{k}^{b} v_{2 m t} \mathrm{~d} C_{m t}\right) \\
\vdots \\
\left(\int_{a}^{b} v_{n 1 t} \mathrm{~d} C_{1 t}+\int_{a}^{k} v_{n 1 t} \mathrm{~d} C_{1 t}\right)+\cdots+\left(\int_{a}^{b} v_{n m t} \mathrm{~d} C_{m t}+\int_{k}^{b} v_{n m t} \mathrm{~d} C_{m t}\right)
\end{array}\right) .
$$

It follows from Theorem 17 that

$$
\int_{a}^{b} v_{t} \mathrm{~d}_{t}=\int_{a}^{k} v_{t} \mathrm{~d} \mathbf{C}_{t}+\int_{k}^{b} v_{t} \mathrm{~d}_{t} .
$$

The proof is completed.

\section{Conclusions}

The main result in this paper can be summarized as follows: (a) the properties of one-dimensional complex fuzzy integral were deduced, containing the existence of complex fuzzy integral and linear properties of this integral; (b) the definition of $m$-dimensional complex integral was presented; (c) similar properties of multidimensional complex fuzzy integral were obtained.

\section{Conflict of Interests}

The authors declare that there is no conflict of interests regarding the publication of this paper.

\section{Acknowledgments}

This work was supported by Natural Science Foundation of China Grant no. 11201110, no. 61374184, and no. 11101115 and Outstanding Youth Science Fund of the Education Department of Hebei Province no. Y2012021.

\section{References}

[1] B. Liu, "Fuzzy process, hybrid process and uncertain process," Journal of Uncertain Systems, vol. 2, no. 1, pp. 3-16, 2008.

[2] B. Liu, "A survey of credibility theory," Fuzzy Optimization and Decision Making, vol. 5, no. 4, pp. 387-408, 2006.

[3] B. Liu, Uncertainty Theory, Springer, Berlin, Germany, 2004.

[4] C. You, H. Huo, and W. Wang, "Multi-dimensional Liu process, differential and integral," East Asian Mathematical Journal, vol. 29, no. 1, pp. 13-22, 2013.

[5] C. You and G. Wang, "Properties of a new kind of fuzzy integral," Journal of Hebei University (Natural Science Edition), vol. 31, no. 4, pp. 337-340, 2011.

[6] C. You and H. Huo, "Properties of muti-dimensional Liu process," in Proceedings of the 6th International Conference on Business Intelligence and Financial Engineering, Hangzhou, China, November 2013.
[7] W. Dai, "Reflection principle of Liu process," 2007, http://orsc .edu.cn/process/071110.pdf.

[8] C. You, W. Wang, and H. Huo, "Existence and uniqueness theorems for fuzzy differential equations," Journal of Uncertain Systems, vol. 7, no. 4, pp. 303-315, 2013.

[9] X. Chen and Z. Qin, "A new existence and uniqueness theorem for fuzzy differential equations," International Journal of Fuzzy Systems, vol. 13, no. 2, pp. 148-151, 2011.

[10] Z. Qin and X. Li, "Option pricing formula for fuzzy financial market," Journal of Uncertain Systems, vol. 2, no. 1, pp. 17-21, 2008.

[11] J. Gao, Credibilistic option pricing: A new model, 2007, http:// orsc.edu.cn/process/071124.pdf.

[12] J. Peng, "A general stock model for fuzzy markets," Journal of Uncertain Systems, vol. 2, no. 4, pp. 248-254, 2008.

[13] Z. Qin and X. Li, "Expected payoff of trading strategies involving European options for fuzzy financial market," Iranian Journal of Fuzzy Systems, vol. 8, no. 3, pp. 81-94, 2011.

[14] Y. Zhu, "Fuzzy optimal control with application to portfolio selection," 2007, http://www.orsc.edu.cn/process/080117.pdf.

[15] Z. Qin, M. Bai, and D. Ralescu, "A fuzzy control system with application to production planning problems," Information Sciences, vol. 181, no. 5, pp. 1018-1027, 2011.

[16] L. Yang, "Some properties of complex fuzzy variable," Tech. Rep., 2007.

[17] Z. Qin and M. Wen, "On analytic functions of complex Liu process," Journal of Intelligent and Fuzzy Systems, vol. 28, no. 4, pp. 1627-1633, 2015. 


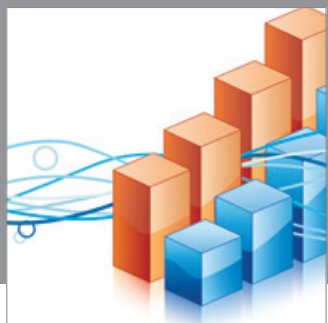

Advances in

Operations Research

mansans

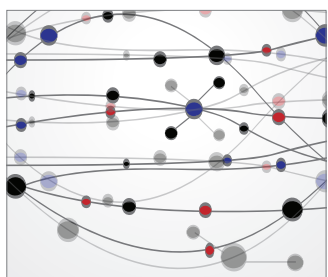

The Scientific World Journal
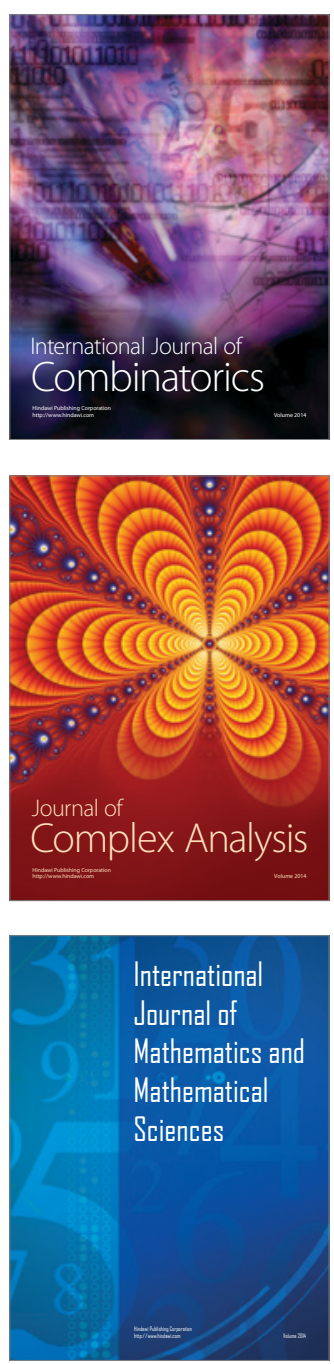
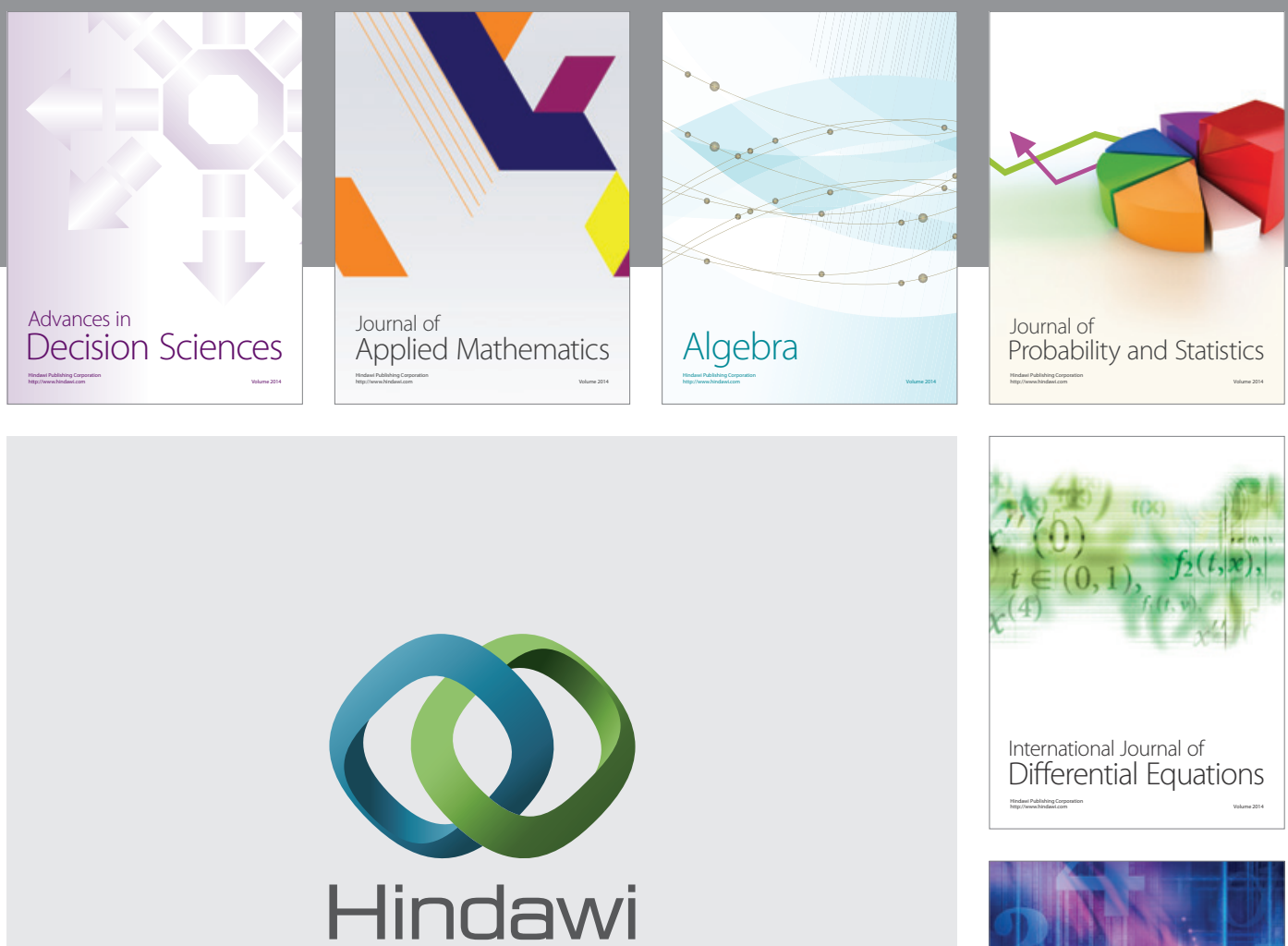

Submit your manuscripts at http://www.hindawi.com
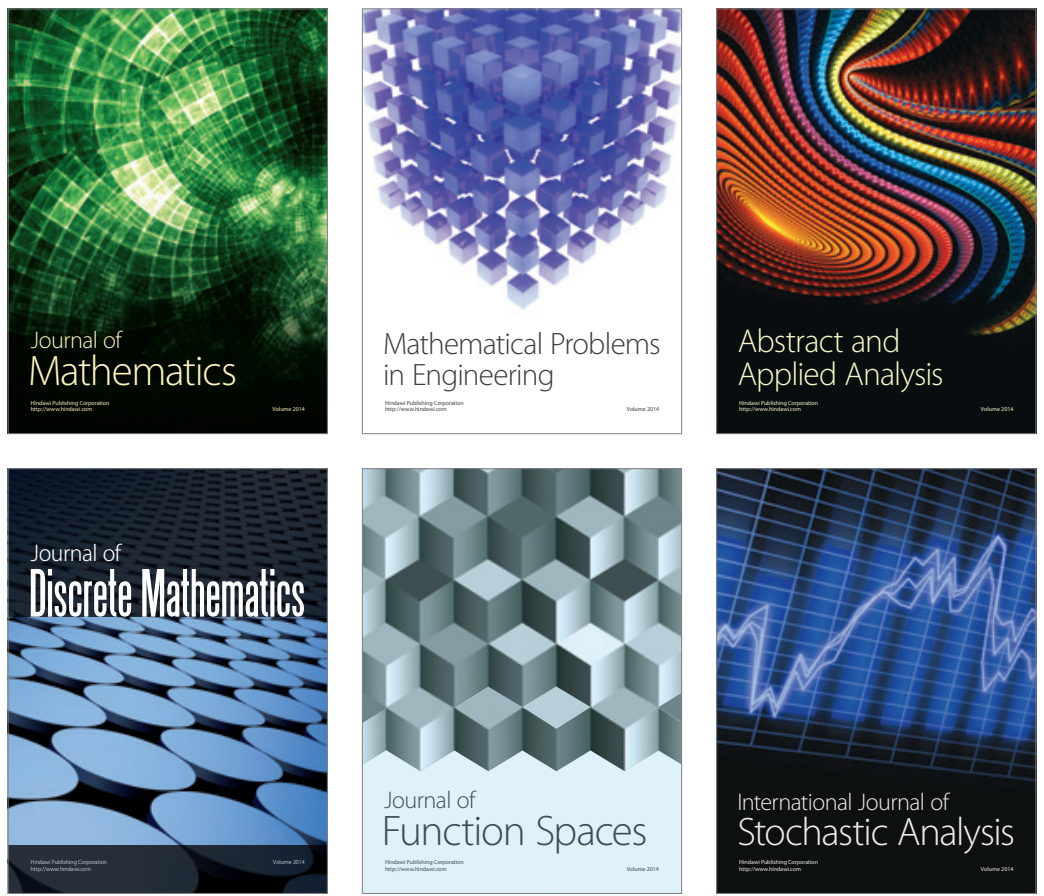

Journal of

Function Spaces

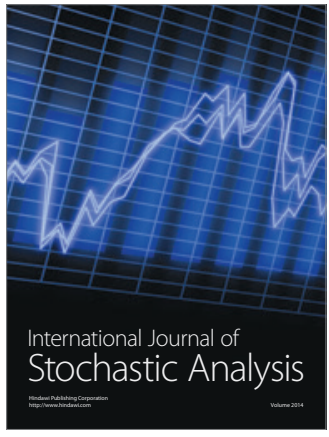

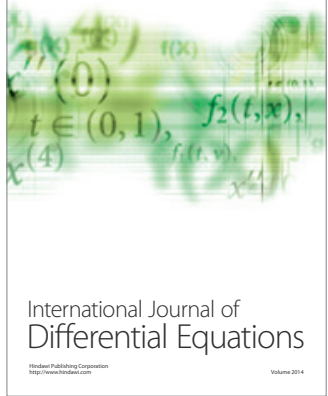
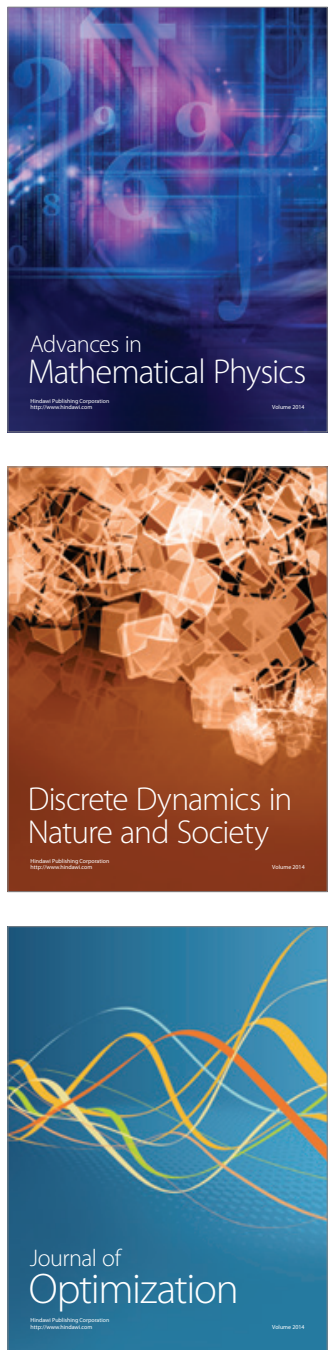